\title{
Gradhiva
}

\section{La channeling zone : religion populaire, État local et rites de légitimation en Chine rurale à l'ère de la réforme}

The Channeling Zone: Popular Religion, the Local State and Rituals of Legitimation in Reform- Era Rural China

\section{Adam Yuet Chau}

Traducteur : Camille Joseph

\section{(2) OpenEdition}

Journals

Édition électronique

URL : http://journals.openedition.org/gradhiva/2499

DOI : $10.4000 /$ gradhiva.2499

ISSN : 1760-849X

Éditeur

Musée du quai Branly Jacques Chirac

Édition imprimée

Date de publication : 1 décembre 2012

Pagination : 156-177

ISBN : 978-2-35744-048-7

ISSN : 0764-8928

Référence électronique

Adam Yuet Chau, «La channeling zone : religion populaire, État local et rites de légitimation en Chine rurale à l'ère de la réforme », Gradhiva [En ligne], 16 | 2012, mis en ligne le 01 décembre 2015, consulté le 02 mai 2019. URL : http://journals.openedition.org/gradhiva/2499 ; DOI : 10.4000/gradhiva.2499 


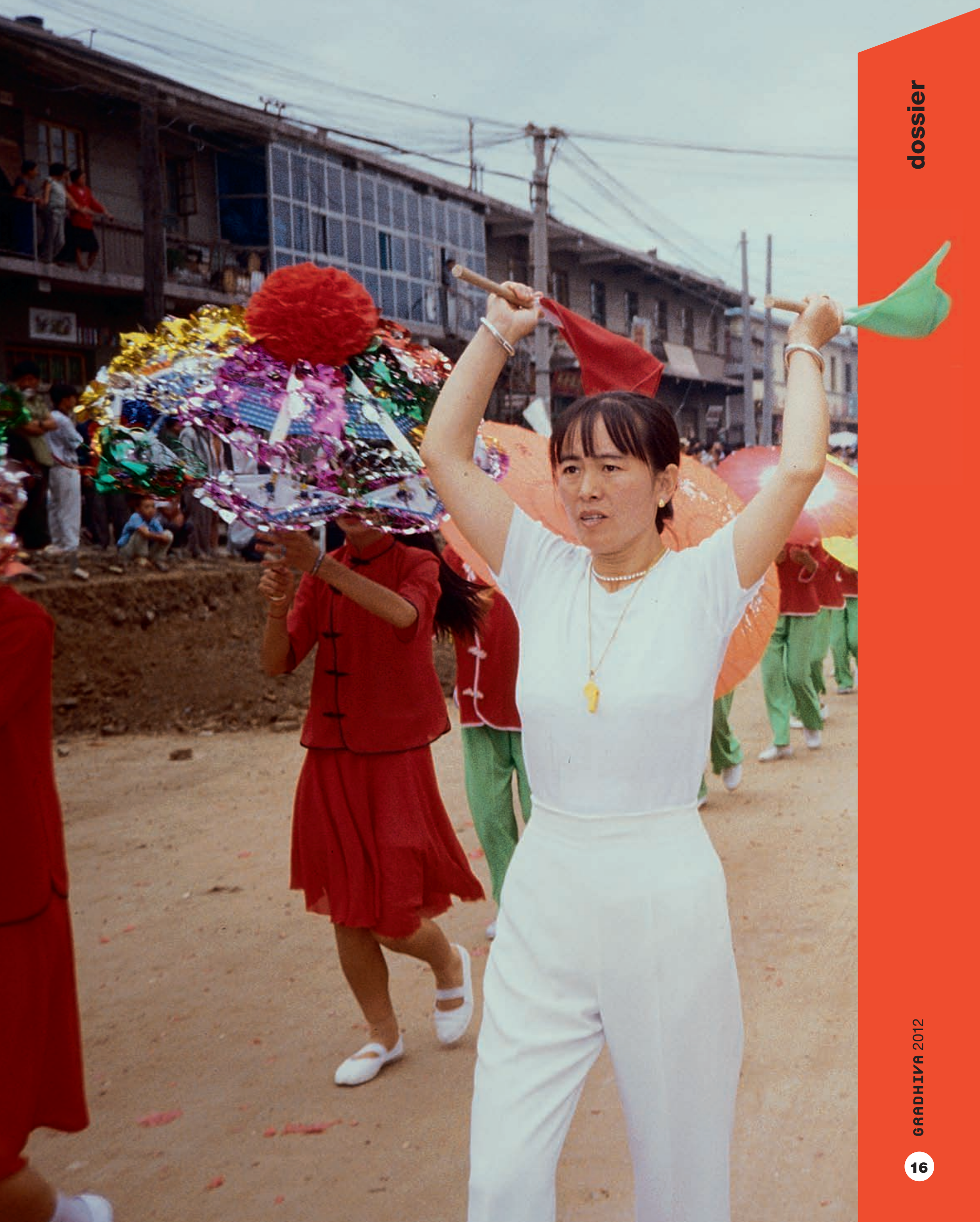




\section{La channeling zone:}

religion populaire, État local et rites de légitimation en Chine rurale à l'ère de la réforme

par Adam Yuet Chau

La politique officielle chinoise en matière de religion considère les activités populaires autour des temples religieux, telles les fêtes de temple, comme des superstitions devant être éliminées. Cependant, durant les trente dernières années - l'époque des réformes -, les leaders de temple se sont localement, dans de nombreuses zones rurales, lancés dans une politique de légitimation afin de protéger, voire d'élargir, leur sphère d'activité. En même temps, la décentralisation du pouvoir politique en Chine a provoqué le développement de pratiques rentières de la part des autorités locales.

Ensemble, ces deux processus ont produit une channeling zone d'avantages opposés autour d'une vaste gamme d'activités (la protection du patrimoine, le commerce, l'éducation, la gestion des forêts, le tourisme, etc.) aboutissant à un renforcement de la légitimité des activités religieuses populaires. Cet article présente le cas d'un temple rural, le temple du Roi Dragon Noir au Shaanbei (dans le nord de la province du Shaanxi), qui met en lumière les rouages d'une telle politique de légitimation. Le processus a atteint son apogée lors d'une “cérémonie de pose d'une plaque» qui eut lieu pendant la fête annuelle du temple et marqua le changement de statut de ce dernier, autrefois lieu de superstition et désormais sanctuaire d'une religion officiellement reconnue. 


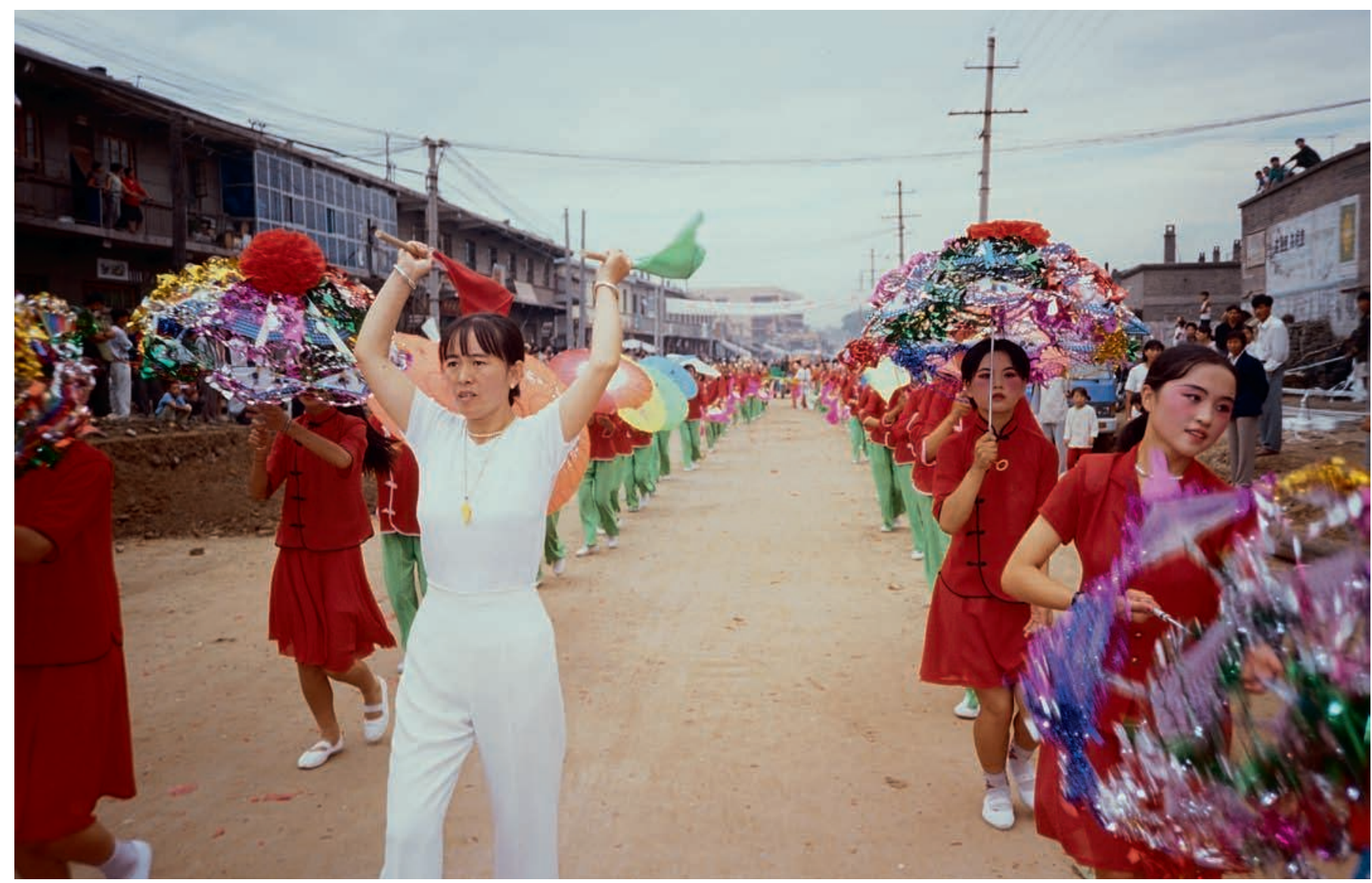

page précédente, ci-dessus et page 177

fig. 1

Danseurs de yangge défilant dans la rue principale de Zhenchuan en direction de la vallée du Roi Dragon, où a lieu le festival du temple, 1998. Photo Adam Chau.

\section{La fête du temple du Roi Dragon Noir}

Nous sommes en 1998. Imaginez que vous êtes un paysan vivant dans un des villages situés sur le célèbre plateau de terre jaune du Shaanbei (au nord de la province du Shaanxi), au nord du centre de la Chine ${ }^{\mathbf{1}}$, et que vous êtes un fidèle de la divinité Roi Dragon Noir (Heilongdawang), I'un des dieux les plus populaires de la région. Imaginez aussi que nous sommes le $13 \mathrm{du}$ sixième mois du calendrier lunaire (ce qui tombe généralement à la mi-juillet), jour anniversaire du Roi Dragon Noir; c'est la journée la plus animée, «rouge et flamboyante» (honghuo), des six jours que dure la fête du temple dans la vallée du Roi Dragon (Longwanggou), à mi-chemin sur la route qui mène au temple du Roi Dragon Noir ${ }^{2}$. Vous vous êtes levé tôt, avez avalé un petit déjeuner rapide, revêtu de beaux vêtements propres; vous vous dirigez maintenant vers le temple à pied, à vélo, sur un cyclomoteur, dans un camion à trois roues ou encore à bord d'un minibus. Le soleil est déjà haut et cette journée de plein été s'annonce chaude. Du matin jusque tard le soir, vous et des dizaines de milliers d'autres fidèles, ainsi que de nombreux touristes piqués par la curiosité - certains auront voyagé pendant de longues heures, voire un jour ou deux -, allez affluer et vous entasser dans l'enceinte du temple pour célébrer les bienfaits et l'efficacité du dieu.

Vous descendez du minibus ou du camion, selon le mode de transport choisi pour vous rendre à la fête. Vous suivez les nuées de fidèles qui remontent la vallée et passez devant des échoppes de nouilles et de pastèques, des 
cercles de parieurs (qui agissent en pleine illégalité, mais sans se cacher), des tentes abritant des spectacles de musique, de danse ou de monstres. Vous achetez quelques paquets d'encens et des monnaies d'offrandes, gravissez les marches abruptes qui mènent au hall principal du temple, jetez les billets dans le grand feu prévu à cet effet, allumez un ruban de pétards; puis vous vous agenouillez en prière devant la statue du dieu Roi Dragon Noir, allumez de l'encens, placez quelques pièces dans l'urne de dons, secouez le cylindre de divination qui prédit votre sort pour les mois à venir et obtenez ainsi le numéro de votre fiche divinatoire, avant d'être immédiatement bousculé par les fidèles qui s'amassent derrière vous. Vous vous dirigez ensuite au fond du hall principal, vers la salle des fiches divinatoires, pour faire interpréter le poème, puis vous prenez place dans la longue file d'attente pour la fontaine magique, où vous remplissez une bouteille de l'eau divine qui guérit afin de la rapporter à un malade dans votre famille, ou bien vous faites accomplir un rituel exorciste par un gérisseur pour que vos enfants soient préservés des malheurs. Ensuite, vous vous frayez un chemin à travers la foule pour assister à un opéra, écouter un conteur, déambuler dans l'enceinte du temple, prendre part à différentes activités, grignoter quelque chose ou avaler un bol de soupe de nouilles épicée, bavarder avec des connaissances, des villageois ou même des inconnus, faire quelques parties de jeu, regarder le feu d'artifice le soir; tout cela en compagnie de dizaines de milliers d'autres participants.

À lire la description de cette fête immensément populaire et riche en sensations, vous, lecteur, pourriez penser que c'est là la preuve que la religion populaire paysanne traditionnelle a miraculeusement survécu à la répression organisée par le gouvernement de la Chine communiste sous Mao et qu'elle est aujourd'hui bien portante et très vivante. Cela supposerait une opposition entre un État séculier soi-disant répressif, d'un côté, et, de l'autre, des communautés locales qui auraient toujours résisté à de telles répressions étatiques. Cette opposition binaire entre communautés locales et État, bien qu'elle soit communément évoquée dans les analyses journalistiques et universitaires sur les relations entre l'État et la société en Chine, est bien trop simpliste et extrêmement insuffisante pour rendre réellement compte des dynamiques sociopolitiques sur le terrain, qu'il s'agisse des questions religieuses ou de tout autre aspect de la vie en Chine. À bien des égards, la religion populaire en Chine rurale, il est vrai, a survécu à la période maoïste et connu un nouvel essor au moment des réformes, mais ce réveil a bien souvent résulté d'une adhésion concertée avec l'État plutôt que d'une résistance à ce dernier. Cette collaboration ne concerne pas seulement les décisions politiques de l'État central en matière de religion et la réaction de la population locale à celles-ci; elle vise avant tout les actions de l'État local, les revendications de pouvoir des élites locales, ainsi que les compromis réciproques, les négociations et les collusions fréquemment observés entre les agents de l'État et ces élites. C'est ainsi que de nombreuses pratiques religieuses populaires classées parmi les activités illégales «superstitieuses » (mixin) - le médiumnisme par exemple, ou encore la distribution et la consommation d'eau magique de guérison, la pratique de rites funéraires, l'usage du fengshui (géomancie) lors des enterrements, les exorcismes, la divination, etc. - ont acquis une légitimité et sont même protégées par la loi. On peut alors parler d'une «politique de légitimation». Dans cet article, prenant pour cas le temple du Roi Dragon Noir, j'étudie ainsi la façon dont
1. La région du Shaanbei s'étend entre Xi'an, la capitale de la province, à l'extrême sud, et, au nord, la MongolieIntérieure (à la limite du désert d'Ordos). Elle possède deux préfectures, Yan'an au sud et Yulin au nord. Aujourd'hui, le Shaanbei est célèbre pour ses sites touristiques,

" trois jaunes et un sacré " (sanhuang yisheng), à savoir la Terre jaune, le fleuve Jaune, la sépulture de l'Empereur jaune et Yan'an, terre sacrée de la révolution.

$$
\begin{aligned}
& \text { 2. L'origine du temple } \\
& \text { remonte au règne de } \\
& \text { Zhengde, de la dynastie Ming } \\
& \text { (1506-1521). La vallée du Roi } \\
& \text { Dragon est située à un peu } \\
& \text { plus d'un kilomètre au sud de } \\
& \text { la ville de Zhenchuan, dans le } \\
& \text { district de Yulin. Surnommée } \\
& \text { «la petite Hong Kong du } \\
& \text { Shaanbei », Zhenchuan est } \\
& \text { un grand entrepôt pour } \\
& \text { le marché de gros et fait la } \\
& \text { liaison entre le nord-ouest } \\
& \text { et le nord de la Chine. Le sort } \\
& \text { du temple du Roi Dragon } \\
& \text { Noir est profondément lié à } \\
& \text { la puissance commerciale } \\
& \text { de Zhenchuan. Aujourd'hui, } \\
& \text { les nombreux petits } \\
& \text { entrepreneurs capitalistes } \\
& \text { qui font don de grosses } \\
& \text { sommes d'argent au temple } \\
& \text { bénéficient d'importantes } \\
& \text { relations commerciales } \\
& \text { à Zhenchuan. }
\end{aligned}
$$


3. La première fois que j'ai rencontré Lao Wang, en 1996, il avait 54 ans et dirigeait l'association du temple depuis quatorze ans. Bien que la renaissance du temple ait été le fruit d'un effort collectif, le talent de dirigeant, les compétences et la vision de Lao Wang sont en grande partie responsables du succès du temple, l'un des plus célèbres de la région du Shaanbei.

4. L'État local se manifeste sous la forme de bureaux du gouvernement et de bureaucrates exerçant au niveau de la ville, du district ou de la préfecture.

5. Bien que je rejette les catégories de "superstition " et d'" activités

superstitieuses",

j'ai choisi, dans le présent article, d'utiliser ces termes conformément aux désignations officielles chinoises. les dirigeants du temple ont entrepris d'astucieuses négociations auprès de différents fonctionnaires locaux pour doter celui-ci d'une légitimité de plus en plus grande. Les dirigeants du temple et les agents de l'État local se transmettent (channel) régulièrement des ressources, établissant ainsi une vaste "voie d'échange" (channeling zone) reliant l'État et la société locale. Au même moment, les frontières entre le «religieux" et le "politique» se brouillent de plus en plus, car la «politique de légitimation» fait désormais partie de la manière dont les gens, au niveau local, «font la religion» (do religion). Le terrain ethnographique évoqué dans cet article est un exemple de rite de légitimation (une “cérémonie de pose d'une plaque»), qui eut lieu au cours de la fête du temple de Longwanggou en 1998. Le chef du temple, Lao Wang (le «Vieux [M.] Wang»), orchestra magistralement ce rite, auquel participèrent toutes sortes d'acteurs sociaux ${ }^{3}$.

\section{L'État local et la politique de légitimation}

Toute analyse du réveil de la religion populaire en Chine rurale doit prendre en compte la distinction fondamentale entre les deux dimensions de l'État socialiste chinois: l'État-parti centralisé qui prend les décisions politiques, d'un côté, et, de l'autre, l'État local qui applique ces décisions et enfreint souvent les réglementations. Pendant la réforme, l'État local a développé de manière considérable son autonomie administrative et fiscale face à l'État central. Au cours des dernières années, les chercheurs sur la Chine ont tenté de regarder au-delà de l'État-parti central et d'analyser les réactions de l'État local (voir par exemple Blecher et Shue 1996; Esherick 1994; Oi 1989, 1999; Shue 1995; Wank 1995, 1999). Ses intérêts étant inscrits dans l'espace local, l'État local agit nécessairement de manière différente de l'État central ${ }^{4}$. Dans la région du Shaanbei, au moment des réformes, les relations entre l'État local et les paysans étaient pour le moins tendues. Les paysans y avaient une image invariablement négative des autorités locales, qu'ils percevaient comme des prédateurs plutôt que comme des serviteurs du peuple (voir Gates 1991; Pan 1997). À l'opposé, une myriade de dieux, déesses, médiums, maîtres yingyang (géomanciens) et devins formaient un large secteur fournissant des services efficaces (ling), et bénéficiaient de la confiance et de la fidélité de la population. Cela explique pourquoi des dizaines de milliers de temples furent reconstruits dans le Shaanbei au cours des années 1980 et 1990; l'activité économique liée à cet ensemble de services rituels a alors explosé.

Comment décrire la relation entre pouvoir local et religion populaire dans le Shaanbei - et, plus généralement, dans la Chine rurale? La liberté de culte est protégée par la Constitution de la République populaire de Chine (RPC), qui condamne toutefois la superstition ${ }^{5}$. II revient alors à l'État d'établir une distinction entre les activités à caractère proprement religieux (zhengdang zongjiao huodong) et les "superstitions féodales" (fengjian mixin). Les pratiques religieuses populaires du Shaanbei se situent en grande partie dans la zone de flou qui existe entre la religion légitime et la superstition illégitime (et donc illégale). Après la Libération, les bureaux des affaires civiles des préfectures de Yulin et Yan'an (minzhengju), qui possédaient des branches dans les administrations de district, furent chargés de superviser les questions religieuses de la région. Plus récemment, dans les années 1990, un bureau des affaires religieuses distinct (zongjiaoju) a été créé pour faire 


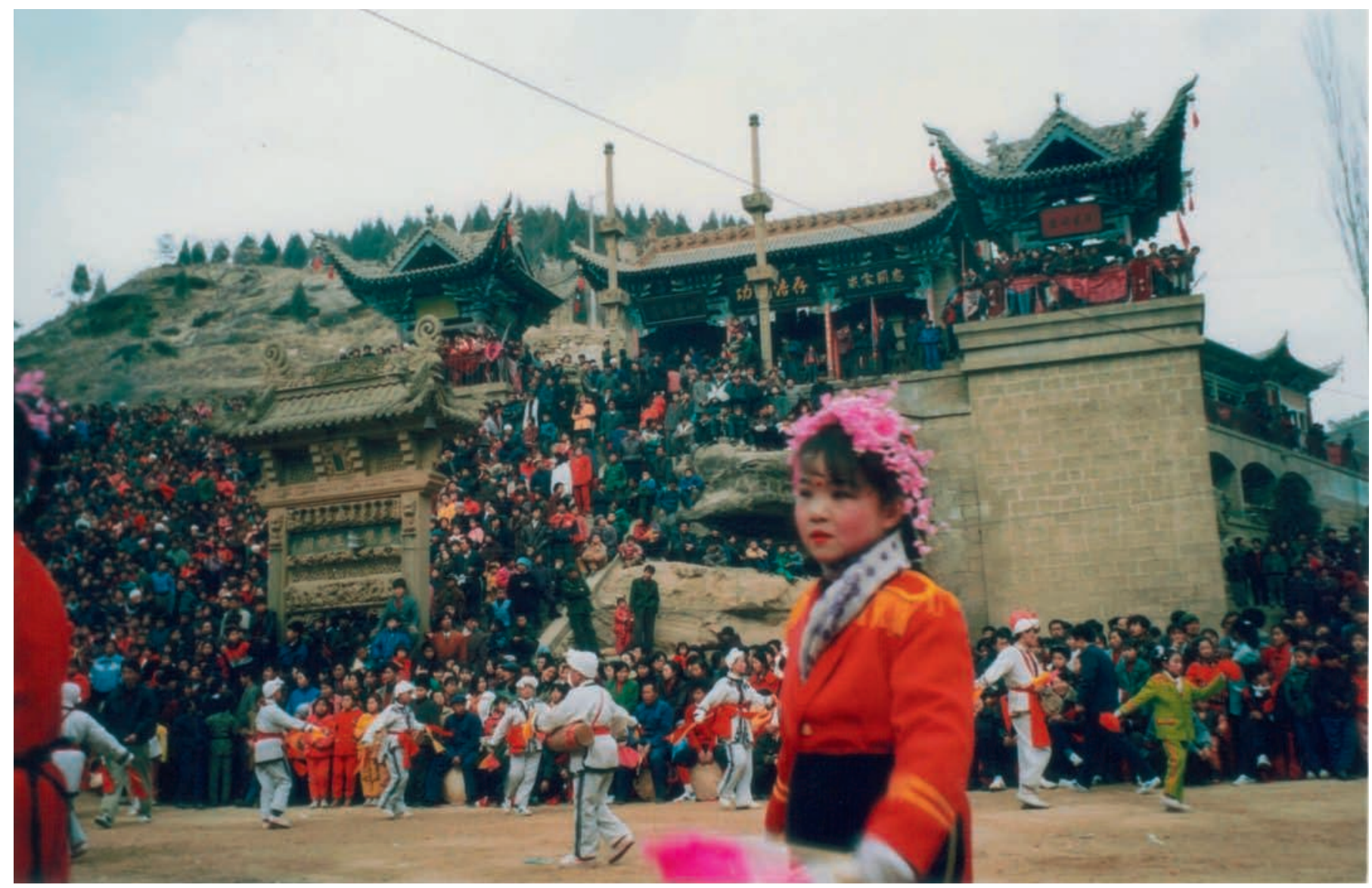

fig. 2

Fête du temple du Roi

Dragon Noir, le sixième mois

du calendrier lunaire, 1998.

Photo Adam Chau. 
6. Le pouvoir local garde toujours un œil sur les pratiques religieuses potentiellement subversives, contre lesquelles il prend parfois des mesures. La répression a notamment touché des mouvements sectaires en pleine résurgence (par exemple Yiguandao), ainsi que le prosélytisme clandestin chrétien. face à la place de plus en plus écrasante prise par la religion. En théorie, ce sont les fonctionnaires du bureau local des affaires religieuses qui sont chargés de faire la distinction entre la religion légitime et les superstitions illégitimes, en application des directives fixées par leurs supérieurs (au sein des bureaux des affaires religieuses de la province) et par le gouvernement central. C'est aussi le bureau qui a pour mission de défendre la religion légale en inscrivant dans les registres et en contrôlant les institutions et le personnel religieux, la police locale ayant, quant à elle, le soin de sévir contre (daji) la superstition. Cependant, dans certains cas, il est difficile de trancher entre la religion à proprement parler et la superstition; d'autre part, ces décisions ne sont pas aisément transposables en termes d'action gouvernementale. À ma connaissance, rien n'a été entrepris, depuis les années 1980, pour cibler les activités superstitieuses dans la région du Shaanbei - un laxisme qui explique notamment la religiosité populaire très vive qu'on peut y observer -, même si une grande partie de ces pratiques (la divination, le médiumnisme, les rituels pour la pluie, le symbolisme de l'enfer et de la rétribution divine, etc.) pourraient, selon les critères de l'époque maoïste, relever de la superstition.

Les critères visant à identifier les superstitions demeurent aujourd'hui les mêmes, mais l'attitude du gouvernement local a changé. Désormais, les agents locaux (c'est-à-dire les responsables des bureaux des affaires religieuses et des bureaux de la sécurité publique) n'ont plus intérêt à prendre des mesures à l'encontre de la superstition car ils n'en retirent aucun bénéfice. À la différence de ce qui se passait sous l'ère maoïste, faire montre d'un zèle ardent (jiji) dans l'éradication des superstitions n'est plus une marque de droiture et d'efficacité politiques (zhengzhi biaoxian). En réalité, la ferveur de la lutte contre la superstition est à ce point liée, dans l'esprit de la population, au comportement d'ultra-gauche propre à l'époque de la Révolution culturelle (qui a été condamnée officiellement comme une aberration et une erreur) qu'elle entache la réputation politique de celui qui en fait la démonstration. Tout le monde au sein de l'État local est bien conscient que la répression de la religion populaire, que cette dernière ait l'apparence d'une superstition ou non, provoquerait une désapprobation générale, voire une réaction de résistance; cela «ne gagnerait pas le cœur des gens ${ }^{6}$ » (bude renxin).

Ce passage d'une attaque radicale contre la tradition à une réglementation paternaliste se manifeste particulièrement bien dans la procédure d'enregistrement des temples auprès du bureau des affaires religieuses de la préfecture. En théorie, seuls les temples légitimement taoïstes ou bouddhistes peuvent devenir membres institutionnels de l'association taoïste ou bouddhiste officielle et nationale. Cependant, dans la pratique, il est très difficile de mesurer avec certitude le caractère strictement taoïste ou bouddhiste des différents temples du Shaanbei. La très grande majorité d'entre eux ne possède pas de clergé ou de doctrine facilement identifiable. D'autre part, chacun présente un éventail de pratiques religieuses parfois très large, ce qui complique l'identification de caractéristiques purement taoïstes ou bouddhistes. Historiquement, les temples taoïstes et bouddhistes ont toujours intégré des éléments «impurs». Ainsi, la plupart des temples du Shaanbei sont, pour reprendre le terme utilisé par les spécialistes des religions chinoises, des temples de religion populaire (folk or popular religious 
temples) offrant un mélange de pratiques diverses qui puisent leurs origines dans des traditions variées. En outre, au problème du syncrétisme taoïste et bouddhiste, apparemment indéchiffrable, s'ajoute la présence, dans de nombreux temples, de pratiques explicitement "superstitieuses» telles que le médiumnisme, qui est non seulement condamné par le bureau des affaires religieuses, mais aussi par les associations taoïstes et bouddhistes officielles. En dépit de ces difficultés apparentes, le processus d'inscription au registre des temples a battu son plein dans les années 1990, sans doute pour répondre à la démultiplication rapide de ces édifices pendant plus d'une dizaine d'années. À cet égard, un représentant du bureau des affaires religieuses de la préfecture de Yulin a reçu, en 1999, le titre de travailleur modèle de la province pour son travail remarquable; cela souligne le fossé qui s'est creusé avec l'ère socialiste, où un cadre aurait été félicité pour avoir fait fermer un grand nombre de temples, non pour avoir procédé à leur inscription officielle.

Cependant, la reconnaissance officielle qui s'obtient par l'inscription au registre est un processus toujours long, qui réclame un fort investissement de la part de la population locale. Ainsi, les représentants officiels de l'État local travaillant pour le bureau des affaires religieuses ou d'autres agences étatiques doivent être reçus comme des invités d'honneur par l'association du temple lors des fêtes, des banquets et des grandes occasions 7 . Les membres du commissariat de police local, conviés afin de maintenir la sécurité et l'ordre, sont rétribués en argent, en cigarettes, en alcool, ou encore logés et nourris gratuitement. II n'est alors pas surprenant que la police s'abstienne de sévir contre les activités des temples. De la même manière, de nombreuses agences étatiques locales tirent d'immenses bénéfices de leurs relations avec les temples, en particulier les plus grands et les plus riches. L'État local et les temples ont dès lors développé une relation de patron à client: les représentants officiels soutiennent les temples qui, en retour, leur montrent un certain respect et leur versent un tribut $\mathbf{8}$.

La nouvelle relation que l'État local entretient avec la société ne concerne pas seulement des questions de réglementation. Elle se caractérise également par une dépendance mutuelle sur le plan pratique. En inscrivant les temples dans leurs registres et en transformant par conséquent les centres de cultes "superstitieux» en «lieux de pratiques religieuses» respectables et officiels pour le culte bouddhiste ou taoïste (zongjiao huodong changsuo), l'État fait acte de complaisance; ces temples sont ainsi protégés contre toute future campagne de lutte contre les superstitions que serait éventuellement amené à lancer l'État central. II est intéressant de noter à ce stade que si, par le passé, le gouvernement impérial tenta de contrôler la religion en accordant fréquemment des titres très pompeux à des divinités individuelles (par exemple Mazu ou Guandi; voir Watson 1985), l'État séculier ne peut aujourd'hui avoir recours à la même stratégie. Mais en inscrivant les temples pour pouvoir les réglementer au besoin, l'État moderne fait appel à une stratégie de contrôle qu'utilisait déjà l'ancien État impérial ${ }^{9}$.

Une autre manière de protéger les pratiques superstitieuses illégales est de les présenter comme des activités désuètes et des «pratiques populaires » inoffensives (fengsu xiguan) appartenant à la «culture traditionnelle»
7. Bien que le bureau des affaires religieuses soit chargé de recueillir les candidatures des temples et d'accorder le statut officiel à ceux qui obtiennent la qualification, ses décisions doivent être entérinées, au niveau de la préfecture, par le gouvernement, le comité du Parti communiste, le Congrès national populaire et la Cour populaire suprême.

8. Le chercheur en sciences politiques, Jean Oi, qualifie les relations croisées de type patron-client entre la société et les agences étatiques locales de "corporatisme local d'État » (voir Oi 1999). On pourrait également définir les temples comme "l'arrière cour économique " de l'État local (voir Lin et Zhang 1999).

9. C.K. Yang (1961: 188) a montré que, sous la dynastie Qing, 84 \% des temples et des monastères recensés avaient vu le jour sans autorisation officielle, chiffre qui suggère un contrôle gouvernemental plutôt faible sur la construction des édifices religieux, à l'instar de ce qui se passe actuellement dans la région du Shaanbei. 


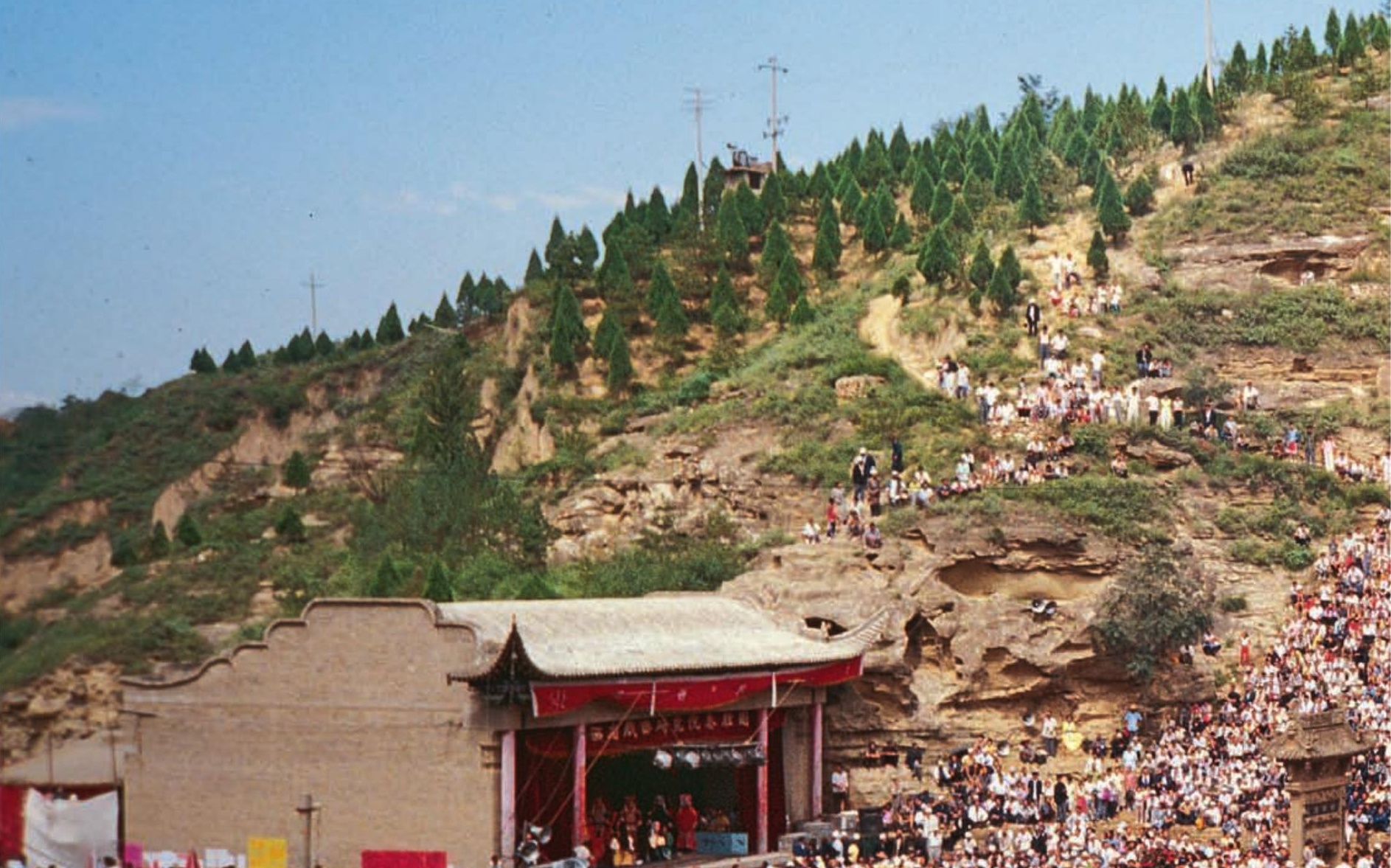

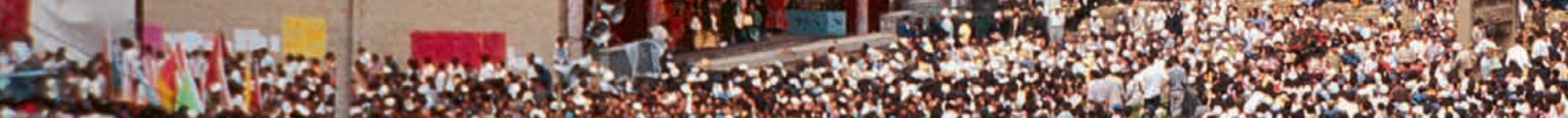

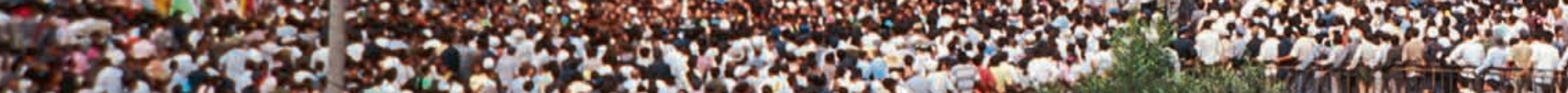

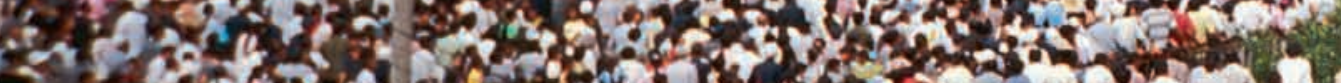

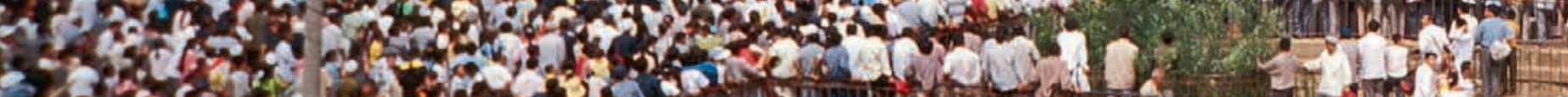

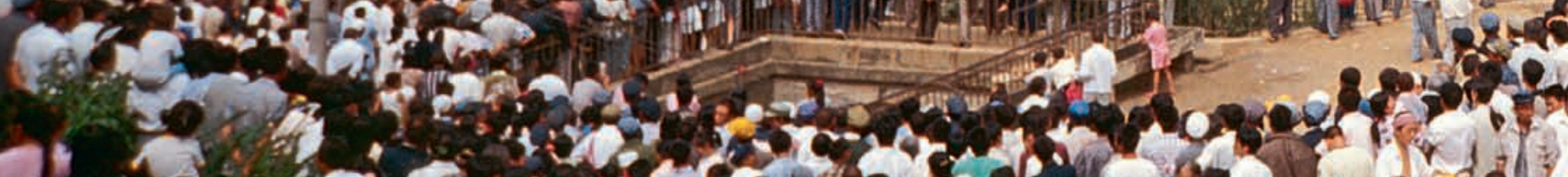

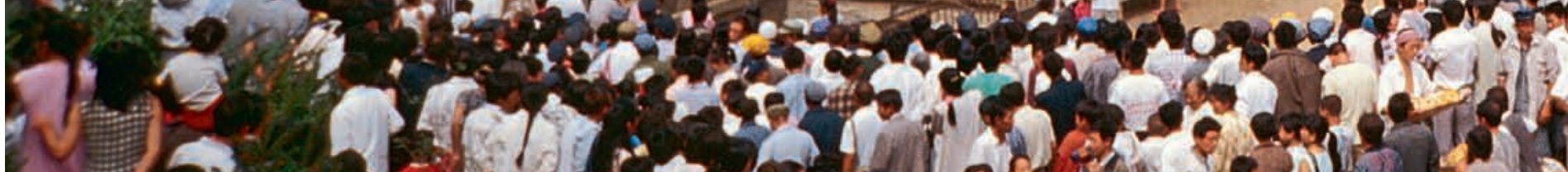

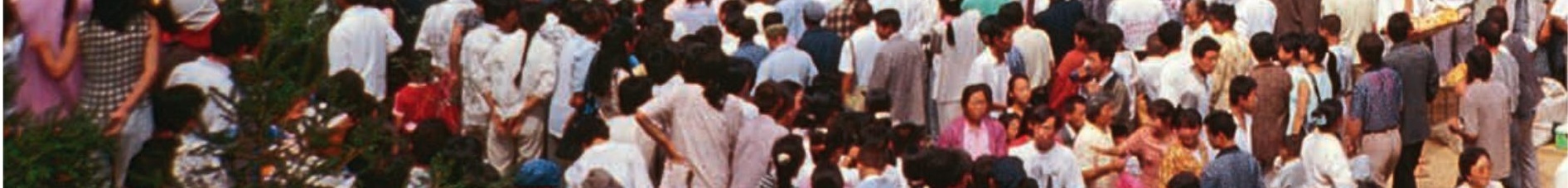

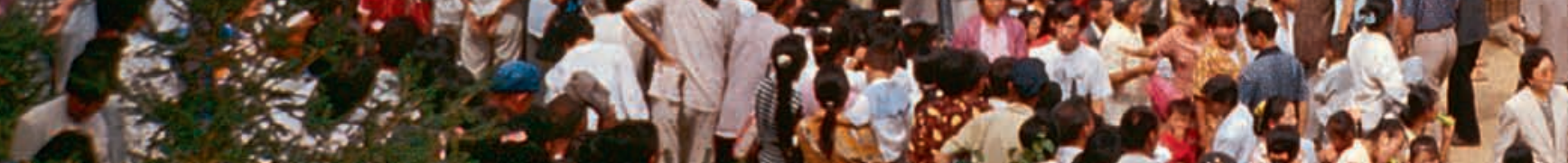

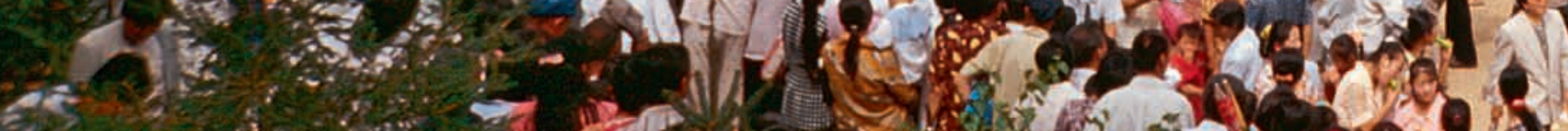
- 7 ir 


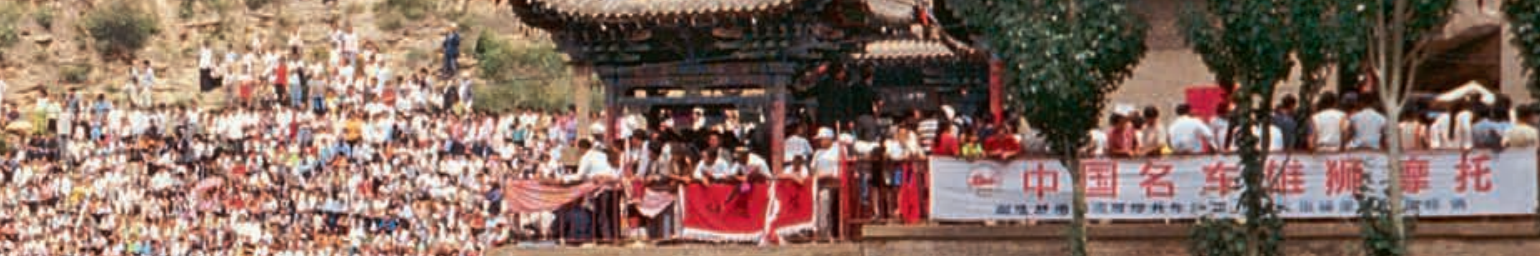

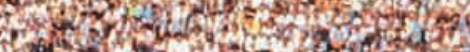

atos.

Whe

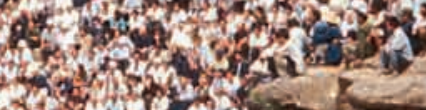

(6)

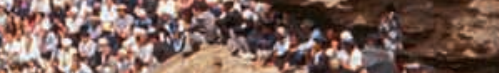

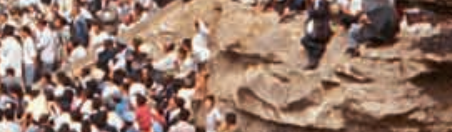

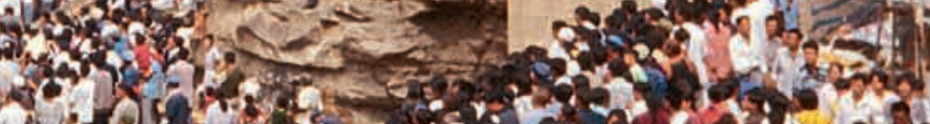

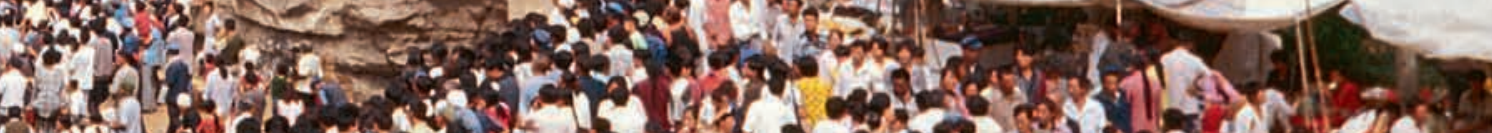

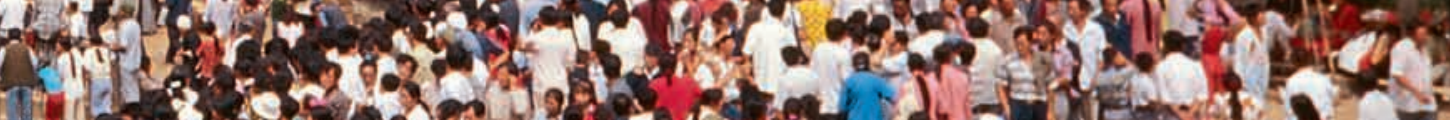
S.

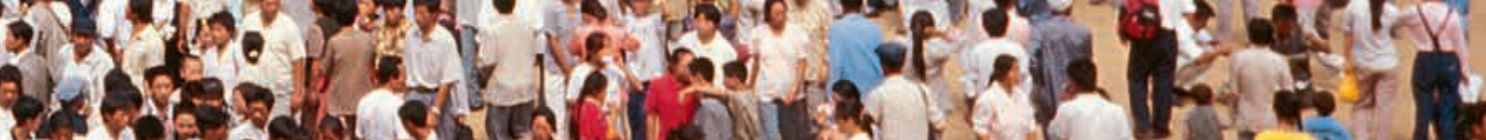

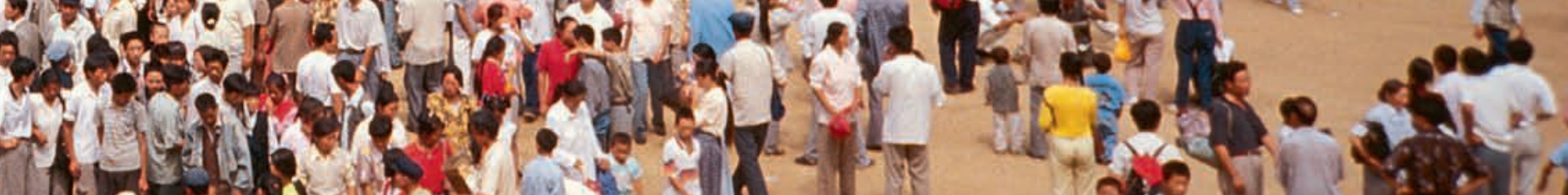
1 . 11
-

$$
\begin{aligned}
& \text { This } \\
& 1140 \text { / }
\end{aligned}
$$

metio 
double page précédente

fig. 3

Le festival au Temple du Roi Dragon Noir.

Sur la gauche, la scène

de l'opéra fait face

au temple, 1998.

Photo Adam Chau. (chuantong wenhua) et, à ce titre, ne justifiant pas les efforts du gouvernement pour les détruire. Ces coutumes, bien que «primitives» et «risibles», peuvent servir d'attractions régionales et culturelles hautes en couleur pour les touristes. Profitant de l'essor de l'industrie touristique, le Shaanbei a, ces dernières années, pris le train en marche afin de promouvoir et de vendre les charmes de sa culture paysanne et la beauté de ses paysages. Des images du Shaanbei rustique ont fait leur apparition à la télévision, dans la presse, dans la littérature et au cinéma. Les folkloristes locaux se sont mis à chanter les louanges de la région. Et des chercheurs viennent de très loin pour étudier la culture populaire locale. Cependant, les temples doivent être vigilants et se protéger contre tout empiètement excessif de la part des bureaux de tourisme récemment créés dans certains États.

L'attention que les chercheurs ont accordée aux traditions populaires participe, dans une très large mesure, de la légitimation de celles-ci. À Taïwan et Hong Kong, la religion populaire a fait son entrée dans l'univers savant depuis longtemps; ce processus de légitimation est souvent directement lié à la formation d'identités régionales (voir par exemple Katz 2003a, 2003b; Sangren 2003). Un mouvement semblable a commencé en Chine, plus particulièrement au Fujian et au Guangdong. Kenneth Dean (1998: 261-263) a livré un compte rendu des «colloques des dieux" au Fujian, où chercheurs, folkloristes locaux, praticiens des religions populaires, représentants des pouvoirs locaux et fidèles se rassemblent lors de manifestations scientifiques dédiées à des divinités ou des traditions religieuses locales. Ce type de collaboration et de collusion n'a fait qu'augmenter en ce début de $x \mathrm{xl}^{\mathrm{e}}$ siècle, car de nombreux temples jouent des coudes pour que les agences étatiques les classent dans le patrimoine culturel immatériel (feiwushi wenhua yichan).

La permissivité dont fait preuve l'État local à l'égard de la religion populaire peut s'expliquer par un autre facteur: les cadres locaux redouteraient les conséquences qui surviendraient s'ils venaient à offenser les dieux. En principe, tous les cadres sont membres du Parti communiste et athées, ils ne craignent ni les dieux ni la vengeance divine. En réalité, cependant, ils appartiennent à des communautés composées en grande majorité de croyants (très souvent des membres de leur propre famille), et sont dès lors eux-mêmes croyants et peu disposés à intervenir dans la réglementation des pratiques religieuses. Un grand nombre de chefs de temple sont d'actuels ou d'anciens secrétaires du Parti de leur village, certains expiant le péché d'avoir fait du tort à des divinités locales sous l'ère maoïste. Dans les analyses qui existent sur la relation entre l'État et la religion populaire, les auteurs se sont abstenus de décrire l'attitude de l'État local (Anagnost 1987, 1994; Bruun 1996; Malarney 1996; Weller 1985, 1987). L'État n'a pas d'emprise directe sur la religion populaire; toute action est en fait laissée aux agents de l'État au niveau local (j'utilise parfois le terme «État local» comme raccourci pour me référer à ces agents en tant que collectif). À défaut d'une étude dans le détail, le terme «État» tend à se scléroser. De même, «le peuple» (ou «la communauté») devient lui aussi un terme figé, en particulier quand on le décrit comme «résistant», toujours de manière collective semble-t-il, à l'intervention de l'État dans la vie religieuse. Ni l'État ni le peuple ne sont des acteurs monolithiques; d'autre 


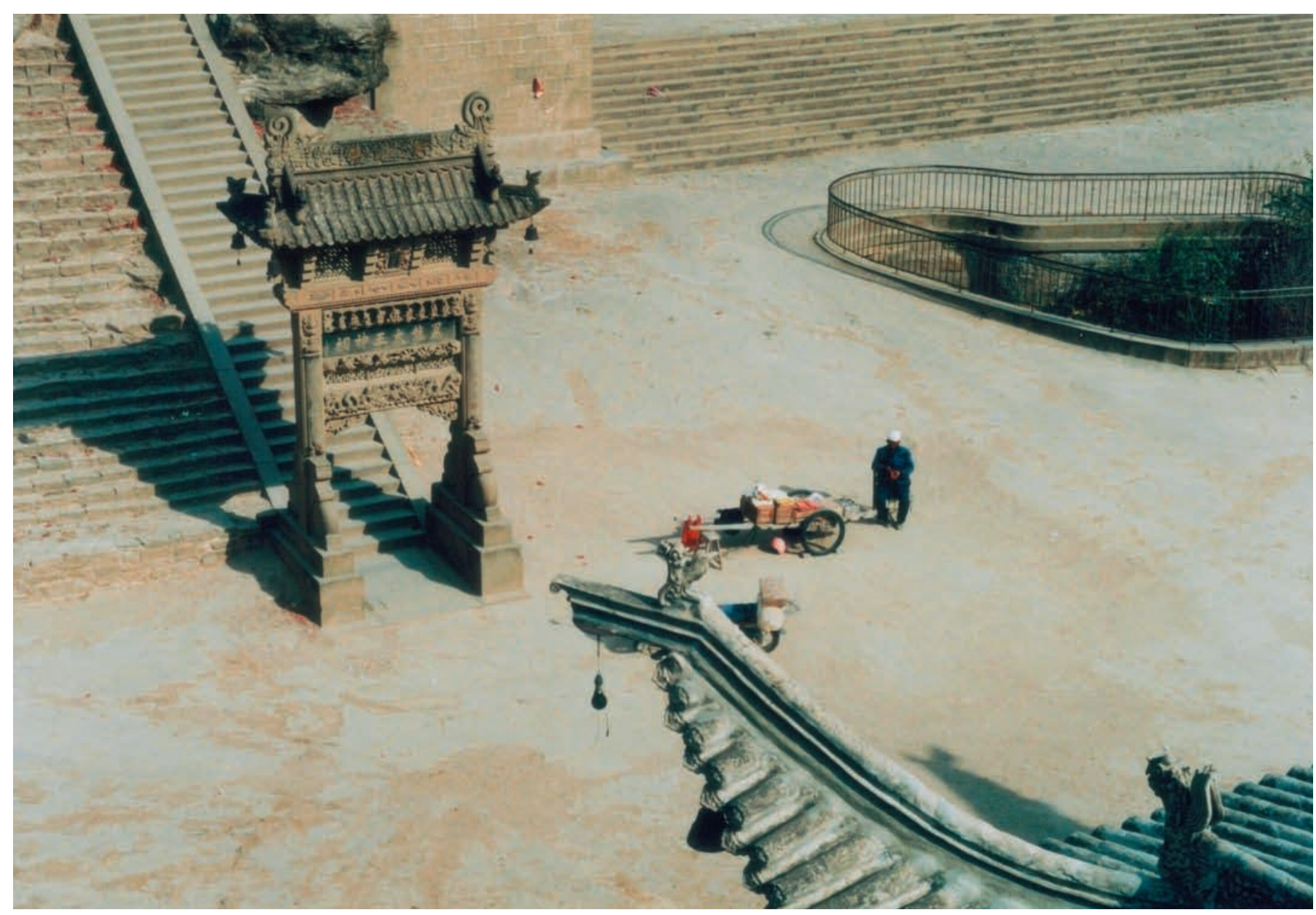

fig. 4

Une paisible journée

de printemps au temple

du Roi Dragon Noir, 1998.

Photo Adam Chau. 
part, la religion populaire implique un nombre important d'acteurs et des actes sociaux très variés.

Depuis sa renaissance au début des années 1980, le temple du Roi Dragon Noir a tissé des relations avec différentes agences étatiques et peu à peu réuni tout l'attirail nécessaire à sa légitimité. En 1982, alors que sa construction venait de s'achever, il reçut la bénédiction du bureau de la culture du district de Yulin (wenhuaju), qui le nomma «trésor culturel du district» (xianji wenwu). Le bureau de gestion du trésor culturel de Longwanggou (Longwanggou wenwu guanlisuo) fut ensuite créé, conférant ainsi officiellement au temple ses premiers galons de légitimité. En 1988, le chef, Lao Wang, et ses collègues financèrent avec le budget du temple un projet de reforestation des collines entourant l'édifice, une stratégie destinée à accroître la légitimité des activités de l'institution. Le bureau forestier de la préfecture de Yulin (linyeju) donna ensuite au projet une appellation officielle: l'arboretum du Vallon de Longwanggou. Ce dernier, grâce à sa dimension écologique, rencontra rapidement un certain écho à l'échelle régionale, nationale et même internationale en tant que premier arboretum civil (non gouvernemental) de Chine. Ce succès a renforcé le statut officiel du temple et augmente sa réputation aujourd'hui encore. En 1996, Lao Wang fonda, toujours à l'aide du budget du temple, l'école primaire de Longwanggou pour remplacer l'ancienne école du village. II lui fallut pour cela emporter l'adhésion du bureau de l'éducation du district de Yulin (jiaoyuju). Toutes les dépenses de l'école, dont les salaires des enseignants, furent prises en charge par le temple, justifiant encore davantage ses activités en dépit de la présence incongrue d'une institution de tradition religieuse dans un projet d'éducation laïque.

Les soutiens officiels mentionnés plus haut, bien qu'ils aient beaucoup compté dans le processus de légitimation du temple du Roi Dragon Noir, se manifestèrent de manière indirecte; aucune de ces aides ne fut directement accordée au temple et à ses activités religieuses en tant que telles. Cependant, en 1998, après de longues années d'attente, bien des retards et des négociations, la récompense arriva enfin: le bureau des affaires religieuses de la préfecture de Yulin accorda le statut officiel à Longwanggou et entérina la création du comité de gestion du temple taoïste de Longwanggou (Longwanggou daoguan guanli weiyuanhui). En d'autres termes, le temple de Heilongdawang était désormais reconnu officiellement comme un temple taoïste et Heilongdawang comme une divinité taoïste. À partir de ce moment, le temple lui-même acquit une légitimité et reçut la protection légale garantie par la constitution de la RPC. En tant que divinités populaires, les rois dragons se situent traditionnellement aux marges du taoïsme officiel; ils ne font pas à proprement parler partie de son panthéon. Mais l'association du temple et le bureau des affaires religieuses étaient prêts à passer outre à ces détails mineurs. La «tao-ification» du temple de Heilongdawang et d'autres édifices comparables les a rendus «identifiables » aux yeux de l'État (Scott 1998), même si ce processus impliqua au passage quelques «erreurs» de lecture. 
Les statuts officiels et les soutiens évoqués ci-dessus relèvent de la stratégie délibérée de dissimulation mise en œuvre par l'association du temple: en effet, ils ont permis de protéger ce dernier en mettant en avant ses aspects culturels, ses réalisations dans le domaine de l'environnement et de l'éducation, ainsi que ses liens avec la religion officielle. En d'autres termes, ces statuts, entérinés par les autorités officielles, ont été savamment utilisés par le temple pour masquer ses autres activités, qui, sans cela, auraient été jugées superstitieuses. Les anthropologues ayant étudié la religion populaire à Taïwan ont noté l'ingéniosité de la population, qui maquille ses rites afin de les rendre acceptables aux yeux d'autorités répressives ou réformistes (par exemple Weller 1987). En Chine, des techniques comparables ont été appliquées pour trouver un compromis entre une soumission complète au contrôle idéologique exercé par l'État-parti (officiellement athée) et une autonomie pleinement revendiquée dans le domaine religieux. Les responsables du temple se sont volontairement pliés à la réglementation étatique dans le seul but de pouvoir conserver une certaine marge de manœuvre. Les agents de l'État local, quant à eux, ont autorisé les activités légales et fermé les yeux sur celles qui ne l'étaient pas. Les deux camps ont trouvé là un terrain d'entente.

\section{La cérémonie de pose d'une plaque commémorant la création} du comité de gestion du sanctuaire taoïste de Longwanggou

La fête du temple du Roi Dragon Noir dure normalement six jours et culmine le treizième jour du sixième mois de l'année, jour anniversaire de la naissance de Heilongdawang. En 1998, un mois avant le début de la fête, le temple reçut l'autorisation de créer le comité de gestion du sanctuaire taoïste de Longwanggou. Lao Wang décida d'organiser une grande cérémonie pour célébrer ce moment important dans l'histoire du temple. Pour la «cérémonie de la pose $\mathbf{1 0}^{\mathbf{1 0}}$ " (guapai yishi), il passa commande à un graveur d'une splendide plaque de bois d'environ deux mètres sur laquelle il fit graver l'inscription suivante: "Comité de gestion du sanctuaire taoïste de Longwanggou » (Longwanggou doaguan guanli weiyuanhui). II convia les responsables officiels concernés et les chefs des autres temples de la région à la cérémonie, qui devait se tenir à midi, le 13 du sixième mois lunaire (le 4 août 1998). De toute la fête du temple, le midi du treizième jour est traditionnellement le moment que l'on juge le plus important. Le choix de l'horaire montre combien Lao Wang prenait l'événement au sérieux. À travers cette cérémonie, il souhaitait littéralement organiser une rencontre entre Heilongdawang, le chef du temple (lui-même), les invités (en particulier les représentants officiels du pouvoir local) et le flot de participants se rendant à la fête. L'autorité séculière de l'État se diffusa ainsi dans l'espace et le temps, renforçant de manière rituelle la légitimité de Longwanggou et de Lao Wang; ce qui n'était alors qu'une fête religieuse populaire se chargea de significations politiques.

Il était presque midi. La poussière jaune qu'avaient soulevée sur leur passage les troupes de théâtre et les danseurs pendant la procession de présentation des offrandes (yinggong) était retombée. Des tables et des chaises avaient été installées sur la scène de l'opéra, face à Heilongdawang et au public. Toute la vallée s'était amassée sur les hauteurs dans le plus grand chaos, cependant que le parterre et les marches de pierre de l'auditorium, où avaient pris place plusieurs milliers de personnes, présentaient un aspect
10. Les plaques sont des marqueurs matériels fondamentaux de la légitimité des institutions dans la culture politique chinoise. Seuls les organes du Parti communiste peuvent utiliser du rouge pour les inscriptions. 


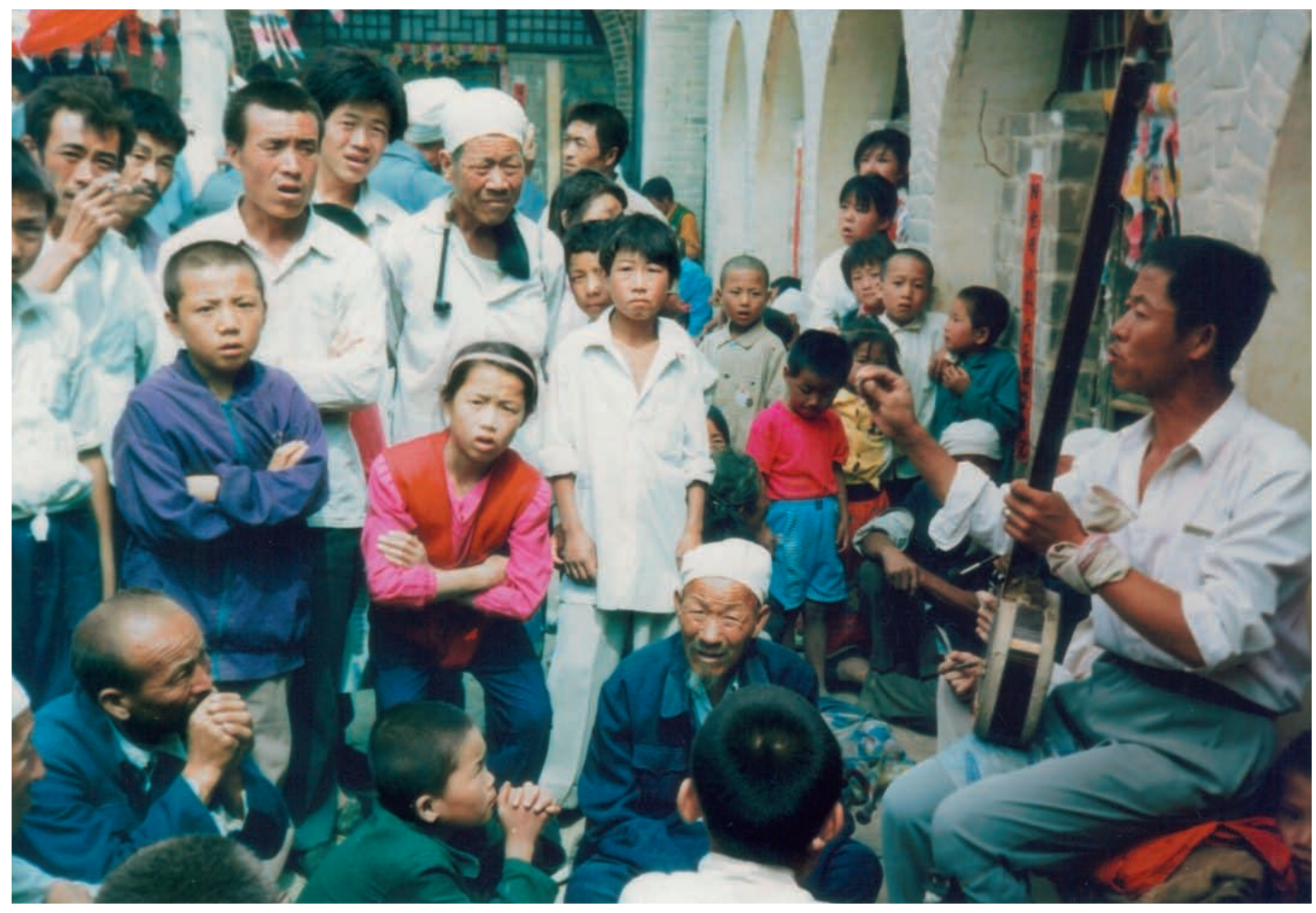

fig. 5

Les communautés

trop pauvres pour s'offrir

des opéras, se divertissent

néanmoins grâce aux

conteurs populaires, 1998

Photo Adam Chau. 
plus ordonné. Partout, un bourdonnement confus se fit entendre. Les hautparleurs annoncèrent que la cérémonie aurait lieu à midi, après quoi l'opéra reprendrait. Une foule grandissante se massa près de la scène pour voir ce qui se passait.

Les invités et les orateurs sortirent des coulisses et s'installèrent les uns après les autres avec les politesses d'usage. Le grand prêtre du sanctuaire taoïste de la Montagne du Nuage Blanc siégea au centre, à la place d'honneur, sans doute parce qu'il était le plus âgé. Le directeur du bureau des affaires religieuses et l'assistant du directeur du bureau des affaires civiles de la préfecture de Yulin prirent place à la gauche du prêtre. Trois chefs de temple, deux représentants de la municipalité, un ancien dirigeant de l'association du temple de Longwanggou et Lao Wang lui-même, qui choisit de s'asseoir à une extrémité (peut-être en signe d'humilité), étaient en rang derrière les longues tables mises bout à bout et recouvertes du tissu rouge cérémoniel. Au milieu se trouvait le micro.

Lao Wang se leva et prononça le premier discours, souhaitant la bienvenue aux «chefs» (lingdao) de l'État local et aux autres invités présents à la fête à l'occasion de la création du comité de gestion du sanctuaire taoïste de Longwanggou. II remercia humblement tous ceux qui avaient apporté leur soutien aux activités du temple et vanta en particulier le succès de l'arboretum. Son discours, qui fut bref, se terminait par ces mots: «ll est de notre devoir, en tant que représentants d'un sanctuaire taoïste, d'appliquer scrupuleusement la politique religieuse du Parti d'“aimer le pays, aimer la religion" (aiguo aijiao) et de promouvoir nos grandes traditions avec exemplarité. " S'ensuivirent des applaudissements, puis on alluma au bord de la scène une longue corde de pétards géants. C'est à ce moment-là qu'on dressa la plaque de bois cachée derrière les invités et qu'on l'amena au-devant de la scène pour la présenter au public. Le chef du bureau des affaires religieuses de la préfecture de Yulin prit le micro: «Je déclare la création officielle du comité de gestion du sanctuaire taoïste de Longwanggou!» Les applaudissements redoublèrent.

On porta l'imposante plaque dans le bureau du temple, où elle fut suspendue à l'extérieur, près de la porte d'entrée, à côté des autres plaques (celles du bureau de gestion du trésor culturel et de l'arboretum); elle semblait ainsi asseoir la légitimité nouvellement acquise par le temple. Pendant ce temps, les invités firent d'autres discours sur la scène de l'opéra. Le grand prêtre du sanctuaire taoïste de la Montagne du Nuage Blanc déclara combien il était heureux que le temple de Heilongdawang fût désormais un autel taoïste «frère». Le chef du bureau des affaires religieuses expliqua qu'il était important d'éliminer peu à peu tous les éléments superstitieux de Longwanggou et de le transformer en un sanctuaire taoïste exemplaire ${ }^{\mathbf{1 1}}$. L'assistant du directeur du bureau des affaires civiles en profita pour promouvoir le nouveau programme caritatif de son organisme. L'un des deux représentants de la municipalité loua Longwanggou pour le rôle qu'il avait joué dans le développement économique de la région. La cérémonie dura en tout et pour tout quarante-cinq minutes. À la fin, la scène fut rapidement évacuée pour laisser place à la représentation de la pièce d'opéra suivante.
11. Pour autant que je sache, Longwanggou n'est pas devenu, après cette cérémonie, plus taoïste qu'il ne l'était auparavant. 
La cérémonie de la pose de la plaque fut, semble-t-il, l'occasion de mettre en scène un événement de plus grande ampleur: non seulement le temple de Heilongdawang reçut ainsi un statut légal officiel et fut désormais identifié comme temple taoïste (et, par suite, protégé par la loi), mais, de manière plus significative, c'est tout le complexe de Longwanggou (comprenant le temple de Heilongdawang, l'arboretum, l'école primaire et le chef du temple lui-même, Lao Wang) qui devint légitime. La cérémonie de la pose, bien que brève et fort simple, fut d'une grande intensité. L'horaire choisi correspondait au moment pendant lequel on estime que Heilongdawang se manifeste avec le plus de force. On fit ainsi coïncider la présence charismatique de Lao Wang avec la présence magique de Heilongdawang et la présence politique du pouvoir local. La cérémonie contribua également à accroître les pouvoirs de chef de Lao Wang.

La cérémonie de la pose de la plaque illustre donc avec pertinence la «Symbolique du pouvoir» (Geertz 1980: 98) à Longwanggou. La légitimité, pour ne rien dire de la légalité, du complexe de Longwanggou (qui inclut la direction exercée par Lao Wang) se manifesta de manière rituelle et fut confirmée par la présence des participants à la fête: Heilongdawang (qui regarde vers le bas depuis son trône, reçoit les offrandes, écoute prières et requêtes), Lao Wang (qui accueille les invités, fait un discours, accroche la plaque, s'attelle à tous les problèmes, petits et grands), les représentants de l'État local (reçus et divertis, qui délivrent des discours, serrent la main d'anciens amis et collègues), les dirigeants des organisations et communautés religieuses voisines (qui discutent entre eux, font des discours) et, surtout, la masse des participants à la fête (qui assistent à l'opéra, écoutent les discours, prient Heilongdawang, jouent à des jeux, vendent et achètent toutes sortes de choses, sont juste là et s'amusent). II régnait une atmosphère d'abondance, de grand divertissement (honghuo) et de «bien-être partagé » (Abélès 1988: 396).

Lao Wang et Longwanggou consacrèrent beaucoup de temps au «travail rituel» (ibid.: 398) et en tirèrent bien des avantages. Bien qu'il ne fût pas un spécialiste religieux, Lao Wang montra à n'en pas douter une grande maîtrise dans l'orchestration rituelle du pouvoir et de l'autorité. Les ressources matérielles et symboliques dont il disposait pour la mise en scène de la cérémonie et du rite de légitimation étaient considérables, même si elles faisaient certainement pâle figure en comparaison de celles qui furent utilisées par l'empereur de Qianlong lors des sacrifices impériaux (voir Zito 1997) ou par l'État-parti chinois lors des cérémonies de la fête nationale du $1^{\text {er }}$ octobre (par exemple celle du cinquantième anniversaire de la RPC en 1999). En tant que chef du temple, Lao Wang pouvait non seulement se targuer d'avoir Heilangdawang à ses côtés, mais également de disposer d'une cagnotte de dons qui, à la fin des années 1990, s'élevait à plus d'un million de dollars chinois chaque année.

La cérémonie de la pose de la plaque fut l'occasion de réunir des intérêts et des projets très différents: l'ambition politique de Lao Wang, la présence de plus en plus bienveillante du pouvoir local et de sa réglementation, et, enfin, la religiosité collective et les envies de divertissement des participants. Chaque acteur social vint avec ses propres motivations et 
donna un sens personnel au rituel de la cérémonie. Étranger à toute considération politique, Lao Wang, qui avait ses rivaux à l'œil, s'assura le soutien de l'État local afin de protéger le temple et de consolider sa position de chef. Parallèlement, il démontrait ainsi aux représentants des autorités locales que le temple de Heilongdawang jouissait d'une grande popularité et que ces derniers auraient tout intérêt à le protéger plutôt qu'à s'immiscer dans ses affaires. Les représentants officiels de l'État local consentirent à se faire les complices de Lao Wang dans son projet, car ils avaient beaucoup à gagner en défendant la bonne réputation d'un site de pèlerinage local. Ces représentants, que le public jugeait de plus en plus corrompus et indifférents, ne furent que trop heureux de manifester ainsi leur présence bienveillante au sein de la population, accordant un soutien paternaliste à tout ce folklore rustique et peut-être quelque peu idiot à leurs yeux.

Le mélange complexe d'éléments relatifs au pouvoir religieux et politique et à l'autorité dans ce rite de légitimation nous amène à conclure, pour reprendre les termes du philosophe Claude Lefort, qu'«on ne saurait séparer ce qui relève de l'élaboration d'une forme politique [...] et ce qui relève de l'élaboration d'une forme religieuse" (Lefort 1986: 261; cité en anglais dans Abélès 1988: 392). De nos jours, les actes politiques prennent des connotations religieuses et des formes rituelles diverses, à l'instar des "drames politiques" auxquels fut confronté François Mitterrand, comme l'a montré Marc Abélès (1988), ou encore des "sociodrames" (sociodramas) étudiés par James McLeod (1999) lors des campagnes présidentielles aux États-Unis. En Chine rurale contemporaine, la résurgence de tout symbolisme religieux dans la vie politique ainsi que l'intervention sciemment planifiée de l'État séculier dans les affaires religieuses populaires indiquent un changement: les anciennes pratiques rituelles de l'État communiste, qui étaient jusqu'alors strictement politiques (voir Apter et Saich 1994; Bennett 1976; Whyte 1974), ont été délaissées au profit d'un amalgame de formes d'expression et d'affirmation du pouvoir et de l'autorité sociopolitiques qui puisent à des sources différentes dans une société en voie de pluralisation.

\section{La channeling zone: les relations changeantes entre l'État et la société en Chine rurale}

À l'ère de la réforme, la vie politique a connu un changement radical par rapport à la période maoïste. Au plan national, la génération la plus ancienne des dirigeants révolutionnaires a laissé place à une nouvelle génération de technocrates, modifiant ainsi les priorités de l'État qui a abandonné les campagnes politiques pour la promotion de la croissance économique. Au plan local, le critère principal pour évaluer la qualité d'un responsable n'est désormais plus la performance politique mais, dans une large mesure, le développement économique. Le cas de Longwanggou montre que l'État local est prêt à assurer la protection des temples en échange d'un profit financier, même si cela implique qu'il faille défendre des activités superstitieuses et techniquement illégales. L'attitude permissive de l'État local est l'un des facteurs principaux expliquant le réveil de la religiosité populaire dans le Shaanbei.

L'histoire de Lao Wang et de Longwanggou met en outre le doigt sur les domaines de recherche pertinents concernant la formation d'élites locales au sein des communautés en Chine rurale sous la réforme. Un de 
12. J'emprunte cette expression au titre du livre de l'anthropologue Michael Brown sur les pratiques New Age du channeling aux États-Unis (voir Brown 1997).

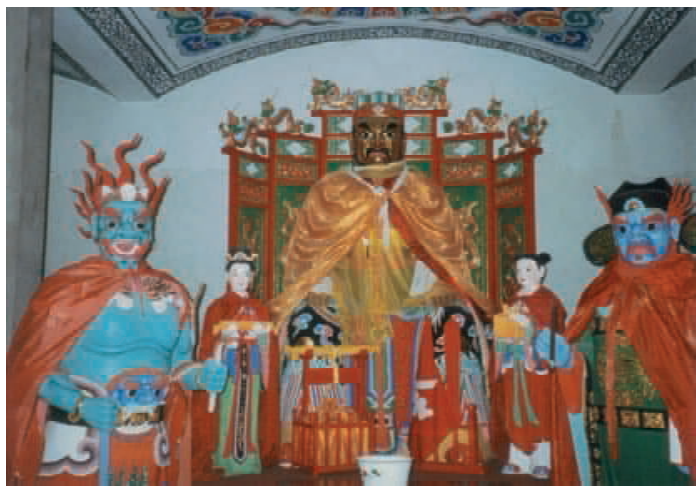

fig. 6

Statue du Roi Dragon Noir, 1998. Photo Adam Chau. ces domaines est la réémergence d'un mode «traditionnel » de structure d'autorité dans la politique rurale locale. Prasenjit Duara (1988) a montré que, depuis le début du $x x^{e}$ siècle, l'État a pénétré dans les couches les plus profondes de la société chinoise locale, à tel point que le «nœud (nexus) culturel du pouvoir» traditionnel a été remplacé par un pouvoir mercenaire, dénué de toute légitimité culturelle. Avec d'autres, il affirme que l'intrusion de l'État communiste dans la société locale paysanne a contribué à supprimer les modes traditionnels de direction assurés par les élites au bénéfice d'un mode de pouvoir socialiste dominé par la classe paysanne pauvre et contrôlé de manière autoritaire par le parti révolutionnaire (voir Chan, Madsen et Unger 1992; Friedman, Pickowicz et Selden 1991; Madsen 1984; Siu 1989). Selon d'autres spécialistes, le fait que l'État socialiste ait pris ses distances par rapport à une critique radicale de tout traditionalisme a laissé de l'espace pour la résurgence de formes plus «traditionnelles» d'autorité et de pouvoir (Anagnost 1994; Feuchtwang 1993, 2000 ; Jing 1996; Wang 1996). Le cas de Lao Wang montre que la période des réformes n'a pas fait resurgir un nœud (nexus) culturel traditionnel de pouvoir, mais plutôt une zone d'interactions complexes entre les agents de l'État local et l'élite locale émergente; bien qu'elle dépende de sa «région locale» (locale), sur laquelle elle fonde son pouvoir, cette dernière cherche des appuis à d'autres niveaux afin de consolider sa légitimité.

Au cours des dix dernières années environ, l'idée de minjian (le civil, le non-gouvernemental) a gagné de la visibilité dans le discours officiel de la RPC. Bien que l'accent reste largement mis sur «le peuple» (renmin) en tant qu'objet de l'action de l'État et de la mission de service des «fonctionnaires", l'attention nouvelle portée au minjian laisse entrevoir une sphère publique élargie au sein de laquelle les citoyens agissent de leur propre initiative (voir Dean 1997, 1998; M.M. Yang 1994). Lao Wang et ses associés ont largement bénéficié de la réputation de l'arboretum du Vallon de Longwanggou, le premier arboretum minjian de Chine. En vertu de sa double qualité de structure minjian et de projet populaire, celui-ci réunit deux des plus importants traits idéologiques de la réforme: la privatisation et la protection environnementale. Longwanggou s'est entouré d'une aura de légitimité qui a déclenché, avec raison, la jalousie des représentants des autres temples.

Les associations de temples sont des organisations minjian nées des besoins des différents secteurs de la société au niveau local (voir Brook 1997). Elles sont une forme condensée et visible du pouvoir de la communauté en un lieu spécifique. Ce pouvoir se manifeste par la magnificence d'un temple et par l'ampleur de ses fêtes. Étant donné que les associations de temples sont parmi les institutions sociales populaires les plus actives, elles ont joué un rôle fondamental dans l'expansion de l'espace culturel et de la sphère publique rurale dans la Chine des réformes (voir Gates 2000). Le cas que j'ai étudié montre aussi que, pour survivre et se développer, elles doivent négocier avec les différentes agences de l'État local et verser des rentes aux représentants officiels afin de bénéficier de leur soutien et de leur protection. En d'autres termes, au fur et à mesure que la sphère publique rurale s'étend, elle est confrontée à un État local de plus en plus présent. 


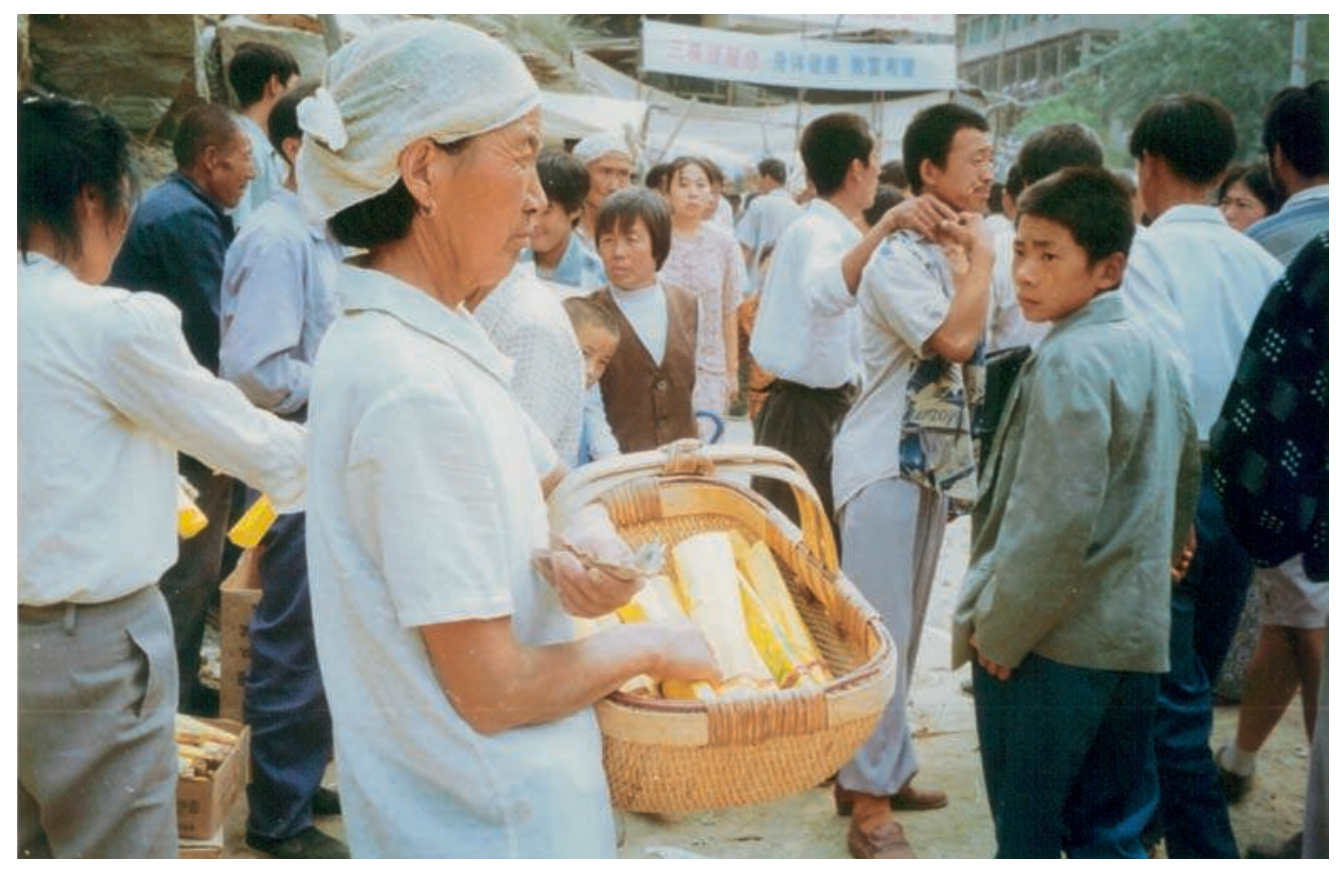

Selon certains spécialistes, l'État chinois facilite autant qu'il contraint le développement de la société civile (Chamberlain 1993; Brook et Frolic 1997). Philip Huang (1993) a utilisé le concept de «troisième espace» pour définir la zone protéiforme qui, prise entre l'État et la société, subit un processus à la fois d'«étatisation » par le haut et de «sociétalisation » par le bas. Les activités religieuses populaires des temples et l'implication de l'État local pour soutenir ces activités représentent une part importante de cet espace dans le Shaanbei actuel (voir Katz 1995: 180-189). La métaphore du channeling est particulièrement adaptée pour décrire les interactions entre l'État et la société locale dans la Chine contemporaine. Comme l'a montré le cas de Longwanggou, l'association du temple interagit non pas avec l'État local en tant que tel, mais plutôt avec les diverses agences locales fonctionnant avec une autonomie relative. Le temple a mis en place différents canaux (channels) de liaison et d'expression permettant de relier l'institution à chaque agence étatique. De la même façon, chaque agence locale a établi des canaux en direction des nombreux solliciteurs présents au sein des institutions locales (c'est-à-dire minjian). Grâce à ces canaux de liaison, les agences étatiques locales font remonter l'argent et les dons vers le haut et, en retour, redistribuent vers le bas des statuts institutionnels officiels et la garantie de leur protection. À l'inverse, les solliciteurs (par exemple les associations de temples) font remonter vers le haut leurs contributions, traitent avec déférence les agences étatiques locales et, en retour, obtiennent la reconnaissance et la légitimité souhaitées. L'individualisation et le poids croissant des structures de l'État local facilitent la démultiplication galopante de ces canaux. L'espace ainsi quadrillé forme ce qu'on peut appeler une channeling zone ${ }^{\mathbf{1 2}}$.

\section{fig. 7}

Femme vendant de la monnaie d'offrande et de l'encens, 1998. Photo Adam Chau.

\section{remerciements}

Je remercie Brigitte Baptandier et Anne-Christine Trémon de m'avoir invité à collaborer à ce numéro spécial de la revue Gradhiva, d'avoir organisé et hébergé la journée d'étude qui s'est déroulée à l'université Paris X-Nanterre en juin 2011, ainsi que d'avoir dirigé ce numéro. Je remercie également Camille Joseph pour la traduction de l'anglais de mon texte. Cet article est issu de mon travail de terrain d'un an et demi dans le Shaanbei, au nord de la Chine, entre 1995 et 1998 (en particulier en 1997 et 1998). Je suis très reconnaissant aux agences et organisations suivantes d'avoir financé mon terrain et la rédaction de mon travail de recherche: la Mellon Foundation, le Center for East Asian Studies de l'université de Stanford, la Wenner-Gren Foundation for Anthropological Research, le Committee on Scholarly Communications with China (au sein de l'American Council of Learned Societies) et la China Times Cultural Foundation. Cet article reprend une partie des éléments évoqués dans mes travaux précédents (voir Chau 2005, 2006, 2008, 2009). 


\section{Abélès, Marc}

1988 «Modern political ritual: Ethnography of an inauguration and a pilgrimage by President Mitterrand ", Current Anthropology 29(3) : 391-404.

\section{Anagnost, Ann S.}

1987 «Politics and magic in contemporary China», Modern China 13(1): 40-61.

1994 «The politics of ritual displacement », in Charles F. Keyes, Laurel Kendall et Helen Hardacre (dir.), Asian Visions of Authority: Religion and the Modern States of East and Southeast Asia. Honolulu, University of Hawai'i Press : 221-254.

\section{Apter, David E. et Saich, Tony}

1994 Revolutionary Discourse in Mao's Republic. Cambridge, Harvard University Press.

\section{Bennett, Gordon}

1976 Yundong Mass Campaigns in Chinese Communist Leadership. Berkeley, Center for Chinese Studies,

University of California ("China Research Monographs»12).

\section{Blecher, Marc et Shue, Vivienne}

1996 Tethered Deer: Government and Economy in a Chinese County. Stanford, Stanford University Press.

\section{Brook, Timothy}

1997 «Auto-organization in Chinese society ", in Brook et Frolic (dir.) [1997]: 19-45.

\section{Brook, Timothy et B. Frolic, Michael (dir.)}

1997 Civil Society in China. Armonk, M.E. Sharpe.

\section{Brown, Michael F.}

1997 The Channeling Zone. American Spirituality in an Anxious Age. Cambridge, Harvard University Press.

\section{Bruun, Ole}

1996 « The fengshui resurgence in China: Conflicting cosmologies between state and peasantry ", The China Journal 36: 47-65.

\section{Chamberlain, Heath B.}

1993 "On the search for civil society in China ", Modern China 19(2): 199-215.

\section{Chan, Anita, Madsen, Richard et Unger, Jonathan}

1992 Chen Village under Mao and Deng. Berkeley, University of California Press.

\section{Chau, Adam Yuet}

2005 «Popular religion in Shaanbei North-Central China ", Journal of Chinese Religions 31(2): 39-79.

2006 Miraculous Response: Doing Popular Religion in Contemporary China. Stanford, Stanford University Press.

2008 "The Sensorial Production of the Social ", Ethnos 73(4) : 485504 .

2009 «Expanding the space of popular religion: Local temple activism and the politics of legitimation in contemporary rural China ", in David L. Wank et Yoshiko Ashiwa (dir.), Making Religion, Making the State: The Politics of Religion in China, Stanford, Stanford University Press : 211-240.

\section{Dean, Kenneth}

1997 «Ritual and space:

Civil society or popular religion?", in Brook et Frolic (dir.) [1997]: 172-192.

1998 Lord of the Three in One: The Spread of a Cult in Southeast China. Princeton, Princeton University Press.
Duara, Prasenjit

1988 Culture, Power, and the State: Rural North China, 1900-1942. Stanford, Stanford University Press.

\section{Esherick, Joseph W.}

1994 « Deconstructing the construction of the party-state Gulin county in the Shaan-Gan-Ning border region ", The China Quarterly 140: 1052-1079.

\section{Feuchtwang, Stephan}

1993 " Historical metaphor: A study of religious representation and the recognition of authority ", Man 28(1): 35-49.

2000 "Religion as resistance", in Elizabeth J. Perry et Mark Selden (dir.), Chinese Society: Change Conflict and Resistance. Londres, Routledge: 161-177.

\section{Feuchtwang, Stephan et Wang, Ming-Ming}

2001 Grassroots Charisma: Four Local Leaders in China. Londres, Routledge.

\section{Friedman, Edward, Pickowicz, Paul G. et Selden, Mark (avec Johnson, Kay Ann)}

1991 Chinese Village, Socialist State. New Haven, Yale University Press.

\section{Gates, Hill}

1991 « Eating for revenge

Consumption and corruption under economic de reform », Dialectical Anthropology 16 : 233-249.

2000 "Religious real estate as indigenous civil space", Bulletin of the Institute of Ethnology, Academia Sinica (Special Issue in Honor of Professor Li Yih-yuan's Retirement [1]) : 313-333

\section{Geertz, Clifford}

1980 Negara: The Theatre State in Nineteenth-Century Bali. Princeton, Princeton University Press.

\section{Huang, Philip C.C.}

1993 " "Public sphere"/“ civil society" in China? The third realm between state and society », Modern China 19(2): 216-240.

Jing, Jun

1996 The Temple of Memories : History, Power, and Morality in a Chinese Village. Stanford Stanford University Press.

\section{Katz, Paul R.}

1995 Demon Hordes and Burning Boats: The Cult of Marshal Wen in Late Imperial Chekiang. Albany, State University of New York Press.

2003a «Identity politics and the study of popular religion in postwar Taiwan ", in Paul R. Katz et Murray A. Rubinstein (dir.), Religion and the Formation of Taiwanese Identities. New York, Palgrave Macmillan: 157-180.

2003b « Religion and the state in post-war Taiwan ", The China Quarterly 174: 395-412.

\section{Lefort, Claude}

1986 Essais sur le politique $\left(x I x^{\ominus}-x x^{e}\right.$ siècles). Paris, Seuil.

\section{Lin, Yi-Min et Zhang, Zhanxin}

1999 «Backyard profit centers The private assets of public agencies ", in Jean C. Oi et Andrew G. Walder (dir.), Property Rights and Economic Reform in China. Stanford, Stanford University Press : 203-225.

\section{Madsen, Richard}

1984 Morality and Power in a Chinese Village. Berkeley, University of California Press. 


\section{Malarney, Shaun Kingsley}

1996 "The limits of "state functionalism" and the reconstruction of funerary ritual in contemporary northern Vietnam ", American Ethnologist 23(3) : 540-560.

\section{Mcleod, James R.}

1999 «The sociodrama

of presidential politics:

Rhetoric, ritual, and power in the era of teledemocracy ", American Anthropologist 101(2): 359-373.

\section{Oi, Jean C.}

1989 State and Peasant in Contemporary China: The Political Economy of Village Government. Berkeley, University of California Press.

1999 Rural China Takes Off: Institutional Foundations of Economic Reform. Berkeley, University of California Press.

\section{Pan, Nianying}

1997 Fupin Shouji (Fieldnotes on my Poverty Relief Work). Shanghai, Shanghai Wenyi Chubanshe.

\section{Sangren, Steven P.}

2003 «Anthropology and identity politics in Taiwan: The relevance of local religion », in Paul R. Katz et Murray A. Rubinstein (dir.) Religion and the Formation of Taiwanese Identities. New York Palgrave Macmillan: 253-287.

\section{Scott, James}

1998 Seeing Like a State: How Certain Schemes to Improve the Human Condition Have Failed. New Haven, Yale University Press.

\section{Shue, Vivienne}

1995 «State sprawl:

The regulatory state and social life in a small Chinese city ", in Deborah S. Davis, Richard Kraus, Barry Naughton et Elizabeth J. Perry (dir.) Urban Spaces in Contemporary China: The Potential for Autonomy and Community in Post-Mao China. Cambridge, Cambridge University Press: 90-112.

\section{Siu, Helen F.}

1989 Agents and Victims in South China: Accomplices in Rural Revolution. New Haven, Yale University Press.

\section{Wang, Ming-Ming}

1996 Shequ de licheng: Xicun hanren jiazu de ge'an yanjiu (A Community's Path: a Case Study of a Han Lineage in Xicun).

Tianjin renmin chubanshe.

\section{Wank, David L.}

1995 «Bureaucratic patronage and private business: Changing networks of power in urban China ", in Andrew G. Walder (dir.) The Waning of the Communist State: Economic Origins of Political Decline in China and Hungary. Berkeley, University of California Press: 153-183.

1999 Commodifying Communism Business, Trust, and Politics in a Chinese City. Cambridge Cambridge University Press.

\section{Watson, James L.}

1985 «Standardizing the gods The promotion of T'ien Hou ("Empress of Heaven") along the South China coast, 960-1960", in David Johnson, Andrew J. Nathan et Evelyn S. Rawski (dir.), Popular Culture in Late Imperial China. Berkeley, University of California Press : 292-324.

\section{Weller, Robert P.}

1985 «Bandits, beggars, and ghosts: The failure of state control over religious interpretation in Taiwan ", American Ethnologist 12(1): 46-61.

1987 «The politics of ritual disguise: Repression and response in Taiwanese popular religion ", Modern China 13(1): 17-39.

\section{Whyte, Martin K.}

1974 Small Groups and Political Rituals in China. Berkeley, University of California Press.

\section{Yang, C.K.}

1961 Religion in Chinese Society : A Study of Contemporary Social Functions of Religion and Some of Their Historical Factors. Berkeley, University of California Press.

\section{Yang, Mayfair Mei-hui}

1994 Gifts, Favors, and Banquets: The Art of Social Relationships in China. Ithaca, Cornell University Press.

\section{Zito, Angela}

1997 Of Body and Brush.

Grand Sacrifice as Text/Performance in Eighteenth-Century China.

Chicago, University of Chicago Press.

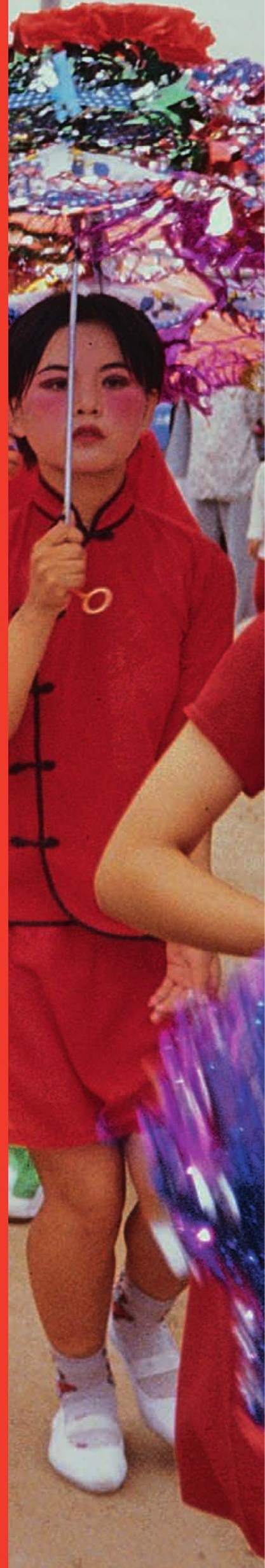

